\title{
A STUDY ON THE TRANSVERSAL OPTICAL MODE IN AMORPHOUS GALLIUM ARSENIDE
}

\author{
M. A. GRADO-CAFFARO* and M. GRADO-CAFFARO
}

Scientific Consultants, C/Julio Palacios, 11, 9-B, 28029-Madrid, Spain

(Received 27 January 1998; In final form 15 April 1998)

Contributions to the far-infrared spectrum corresponding to both dynamical and structural disorders in a-GaAs are examined when frequency coincides with the transversal optical mode. Under these circumstances, dipole moment matrix element is discussed.

Keywords: Far-infrared spectrum; a-GaAs; transversal optical mode; dipole moment matrix element

\section{INTRODUCTION}

Transverse optical mode in amorphous III-V semiconductors presents a great relevance with respect to the far-infrared spectrum corresponding to dynamical disorder; in this context, the frequency associated with this mode is a function of distance. Coincidence of this frequency with the generic frequency has been examined in Ref. [1]. On the other hand, this coincidence has a great importance in the variations of the dipole moment matrix element [2]. Really these subjects are very interesting and the main problems associated with them are still unsolved in a major part at least from the point of view of theory. In the following, we shall try to clarify some of these problems for amorphous gallium arsenide.

\footnotetext{
*Corresponding author.
} 


\section{THEORY}

First of all, consider the expression of the far-infrared spectrum corresponding to dynamical disorder for a-GaAs namely [1, 2, 3, 4]:

$$
\varepsilon_{2}^{(d)}(\omega)=a\left(\varepsilon_{o}-\varepsilon_{\infty}\right) \gamma_{c} \omega_{T O(c)} \omega \int_{o}^{R} \frac{\omega_{T O}^{3}(r) \exp \left(-b r^{2}\right) d r}{\omega_{T O(c)}^{2}\left(\omega_{T O}^{2}(r)-\omega^{2}\right)^{2}+\gamma_{c}^{2} \omega^{2} \omega_{T O}^{2}(r)}
$$

where $\omega$ denotes angular frequency, $a$ and $b$ are positive constants, TO stands for transversal optical mode, $(c)$ denotes crystalline state, $\gamma_{c}$ is the damping factor and $r$ is distance (we consider a one-dimensional model).

Next we will assume that, although $\omega_{T O}$ depends upon distance, the region of dynamical disorder is sufficiently small so that $\omega_{T O}$ can be regarded as approximately constant; structural disorder will be assumed as a considerable disorder so that the total contribution to disorder may be conceived as appreciable. In fact, this situation takes place for example, in a-Ge as a dynamically and structurally disordered material. Under the above conditions for a-GaAs and by taking into account that the dipole moment matrix element is considerably larger for $\omega=\omega_{T O}$ than that for the other frequencies, we get:

$$
\begin{aligned}
\varepsilon_{2}\left(\omega_{T O}\right) & =\varepsilon_{2}^{(d)}\left(\omega_{T O}\right)+\varepsilon_{2}^{(s)}\left(\omega_{T O}\right) \\
& =a\left(\varepsilon_{0}-\varepsilon_{\infty}\right) \gamma_{c}^{-1} \omega_{T O(c)} \int_{o}^{R} e^{-b r^{2}} d r+\frac{\left|\mu\left(\omega_{T O}\right)\right|^{2}}{\omega_{T O}^{2}} \times g\left(\omega_{T O}\right)
\end{aligned}
$$

where $(s)$ denotes structural. On the other hand, $g(\omega)$ is the phonon density of states and $\mu(\omega)$ is the dipole moment matrix element.

Now, for very low frequencies, we have (see, for example, Ref. [1]):

$$
|\mu(\omega)|^{2}=k \omega^{2}\left(\omega \ll \omega_{T O}\right)
$$

where $k$ is a real constant. For $\omega>\omega_{T}$, by Eq. (2) it follows:

$$
\frac{\varepsilon_{2}^{(s)}\left(\omega_{T O}\right)}{g\left(\omega_{T O}\right)}=\frac{\left|\mu\left(\omega_{T O}\right)\right|^{2}}{\omega_{T O}^{2}}>\frac{|\mu(\omega)|^{2}}{\omega^{2}} \neq k
$$


In addition, phonon density of states is given by (see, for example, Ref. [5]):

$$
g(\omega)=(3 N)^{-1} \sum_{n=1}^{n} \delta\left(\omega-\omega_{n}\right)
$$

Since $\delta\left(\omega-\omega_{n}\right)=\delta\left(\omega_{T O}-\omega_{n}\right)$, by formulae (4) and (5), it follows:

$$
\varepsilon_{2}^{(s)}\left(\omega_{T O}\right) \gtrsim \frac{|\mu(\omega)|^{2}}{3 N \omega^{2}} \sum_{n}^{N} \delta\left(\omega-\omega_{n}\right), \quad\left(\omega>\omega_{T O}\right)
$$

\section{CONCLUSIONS}

We have obtained some interesting results for the high-frequency region of the far-infrared range corresponding to a-GaAs; in fact, we have concentrated our attention on the condition $\omega>\omega_{T O}$. At this point, we recall that the magnitude of the dipole moment matrix element for $\omega=\omega_{T O}$ is considerable larger than that for other frequencies [2].

On the other hand, quantitative studies on the high region of the farinfrared margin become useful in order to estimate optical properties of possible optoelectronic devices based upon a-GaAs; in this context, comparison with a-Si and a-Ge is desirable.

\section{References}

[1] Grado-Caffaro, M. A. and Grado-Caffaro, M. (1992). Phys. Lett. A, 169, 399-401.

[2] Mitra, S. S., Paul, D. K., Tsay, Y. F. and Bendow, B. (1974). AIP Conf. Proc., 20 , 284-289.

[3] Grado-Caffaro, M. A. and Grado-Caffaro, M. (1993). Mod. Phys. Lett. B, 7, 1201 1207.

[4] Grado-Caffaro, M. A. and Grado-Caffaro, M. (1994). Mod. Phys. Lett. B, 8, 169172.

[5] Axe, J. D., Keating, D. T., Cargill III, G. S. and Alben, R. (1974). AIP Conf. Proc., 20, 279. 

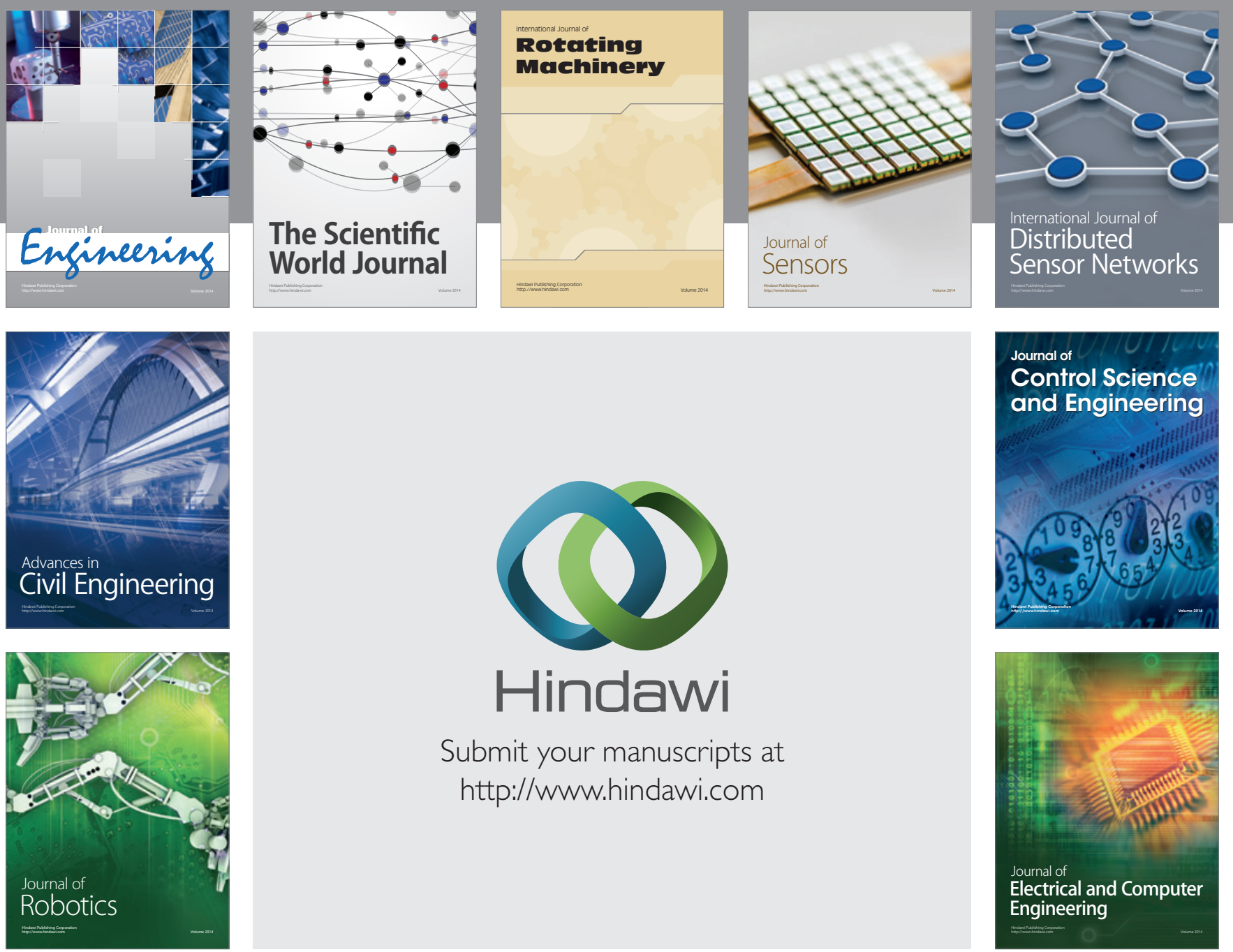

Submit your manuscripts at

http://www.hindawi.com
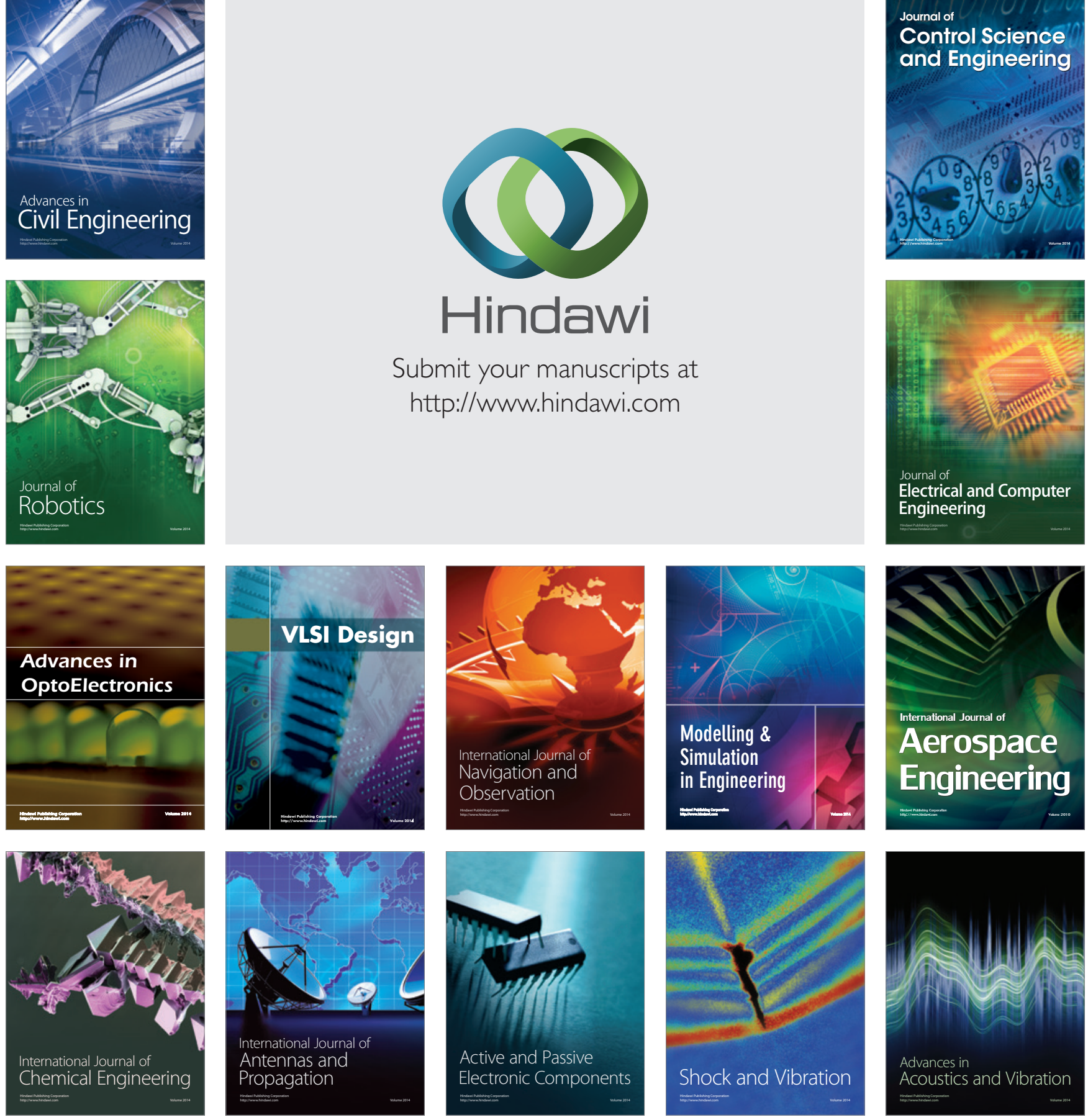